\title{
C-A/AP/\#279
}

July 2007

\section{FFAG-based Accelerator for Radio-Isotopes Production}

\author{
A.G. Ruggiero, J. Alessi, E. Beebe, A. Pikin, T. Roser, D. Trbojevic
}

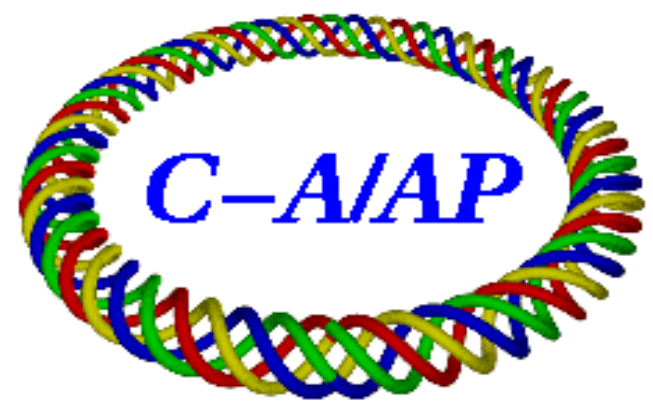

Collider-Accelerator Department Brookhaven National Laboratory Upton, NY 11973 


\title{
FFAG-based Accelerator for Radio-Isotopes Production*
}

\author{
A.G. Ruggiero, J. Alessi, E. Beebe, A. Pikin, T. Roser, and D. Trbojevic \\ Brookhaven National Laboratory \\ June 5, 2007
}

\section{Introduction}

As an alternative to the SuperConducting Linac (SCL) approach [1] we wish to consider also acceleration of ions of Uranium in a pair of Fixed-Field AlternatingGradient (FFAG) accelerators for the production of Radio-Isotopes (RI) and Exotic Nuclear Fragments (ENF). The expectation is that the FFAG accelerators are considerably more economical than SCL accelerators; they involve more conventional magnet and RF technology, and are equivalently reliable and easy to operate.

FFAG accelerators have already been demonstrated half a century ago with the acceleration of electrons [2], and more recently with protons at KEK [3] and KURRI [4] in Japan. The FFAGs in Japan accelerate proton to $150 \mathrm{MeV}$, and involve the use of socalled Scaling Lattice (SL) [5] where chromaticity, that is the variation of the lattice functions with the beam momentum value, is fully compensated with a hyperbolic field profile.

We prefer to adopt here the so-called Non-Scaling Lattice (NSL) with a Linear Field Profile (LFP). This lattice does not compensate for the chromaticity change as in the SL [7]. As a consequence there is a large variation of betatron tunes with beam momentum sweeping across several integral and half-integral resonances during the acceleration cycle. There is of course concern about the beam stability and survival in this situation, but conversely one expects that if the acceleration rate is large enough, say few tens or at most few hundreds revolutions, no harm is done to the beam. Differently from FFAG with SL that have been demonstrated in practice, FFAG with NSL still wait for a practical demonstration.

An important feature of FFAG accelerators (with SL and NSL) is that the bending and focusing magnets are kept at constant fields and gradients, and do not need to be ramped. This simplifies considerably the power supply and vacuum design, and a serious limitation is simultaneously removed on the acceleration rate. Indeed now the acceleration rate is limited by the RF system itself. The rate should be large enough to avoid the problem with the multiple-resonance crossing, and also to boost the average beam power at lower beam average current. The FFAG accelerator can thus be conceived as a Linac entirely bent on itself, on a circular path, with the beam re-circulating in some number of passes.

In the case of acceleration in the low-energy range of heavy particles like protons and, even more, ions of Uranium, the beam velocity varies considerably during the acceleration cycle. For a fast acceleration, frequency-modulated RF cavity system, like that using ferrite, will not do the job well. An alternative is to use broad-band, constant frequency RF cavities as those used in the J-Parc accelerator complex [8]. In this case the $\mathrm{RF}$ frequency is relatively low (few $\mathrm{MHz}$ ), and the voltage of only few tens kVolt per

\footnotetext{
* Work performed under Contract No. DE-AC02-98CH10886 with the U.S. DOE
} 
cavity. Another approach that would allow a considerably higher repetition rate is the method of Harmonic Number Jump (HNJ) [9]. In this case the cavities have also constant frequency in the hundreds $\mathrm{MHz}$ range, and possibly in the $\mathrm{GHz}$ range. But this method requires a programmed energy gain per cavity crossing that can be obtained with an almost linear profile across the radial width of the cavity. The HNJ method needs of course to be demonstrated in practice. Ultimately the HNJ method could be used for a continuous beam mode of operation, since on a given orbit the beam is accelerated by a pre-programmed RF voltage, the profile being kept constant across the with of the cavity at all times, and all orbits can simultaneously be occupied by beam.

\section{The SCL Approach Design}

Let us review briefly the parameters of the SCL design approach [1] so we can make a comparison with those required in the FFAG equivalent design. The proposed layout is shown in Figure 1. It is made of 4 distinct sections joined in a sequence to each other. In the order:

- $\quad$ The Front-End (FE) made of an ECR ion source operating continuously providing a beam of Uranium 238 at two charge states $Q=28$ and 29, at the kinetic energy of $12 \mathrm{keV} / \mathrm{u}$. the beam is pre-chopped and pre-bunched properly before entering the Radio-Frequency Quadrupole (RFQ) that accelerates the beam to $168 \mathrm{keV} / \mathrm{u}$ at the frequency of $57.5 \mathrm{MHz}$.

- The beam enters the Low- $\beta$ Section of the SCL where both charge states $Q=28$ and 29 are simultaneously accelerated to $94.3 \mathrm{MeV} / \mathrm{u}$ by a combination of quarterwavelength multiple-gap cavities at 57.5 and $115 \mathrm{MHz}$. During acceleration the beam bunch length is reduced so it can fit to the RF buckets at higher frequency for better efficiency and larger accelerating gradient. At the exit from the Low- $\beta$ Section the beam hits a thin Lithium film to produce higher charge states in the range $\mathrm{Q}=69$ to 73 .

- The multiple charge state beam enters the Medium- $\beta$ Section of the SCL where it is accelerated to $85.2 \mathrm{MeV} / \mathrm{u}$ with a combination of multiple-gap half-wavelength cavities operating at 172.5 and $345 \mathrm{MHz}$. During acceleration the beam bunch length is reduced further to be effectively squeezed in RF buckets at higher frequency. At the exit of the Medium- $\beta$ Section the beam crosses a second stripping target, made of as a Carbon Wheel, to produce even higher multiple charge states in the range $\mathrm{Q}=88$ to 91 .

- The beam then enters the last High- $\beta$ Section for the final acceleration to 404 $\mathrm{MeV} / \mathrm{u}$ with a sequence of multiple-gap half-wavelength cavities operating at 805 MHz. these cavities have a design similar to that used for the Spallation Neutron Source (SNS) SCL [10]. At the exit of the SCL the beam impinges on the Target proper for the production of RI and ENF. The average beam current on the target is $4.2 \mu \mathrm{A}$-ion. The beam is bunched at $57.5 \mathrm{MHz}$ and there is one bunch every 14 RF buckets at $805 \mathrm{MHz}$. 


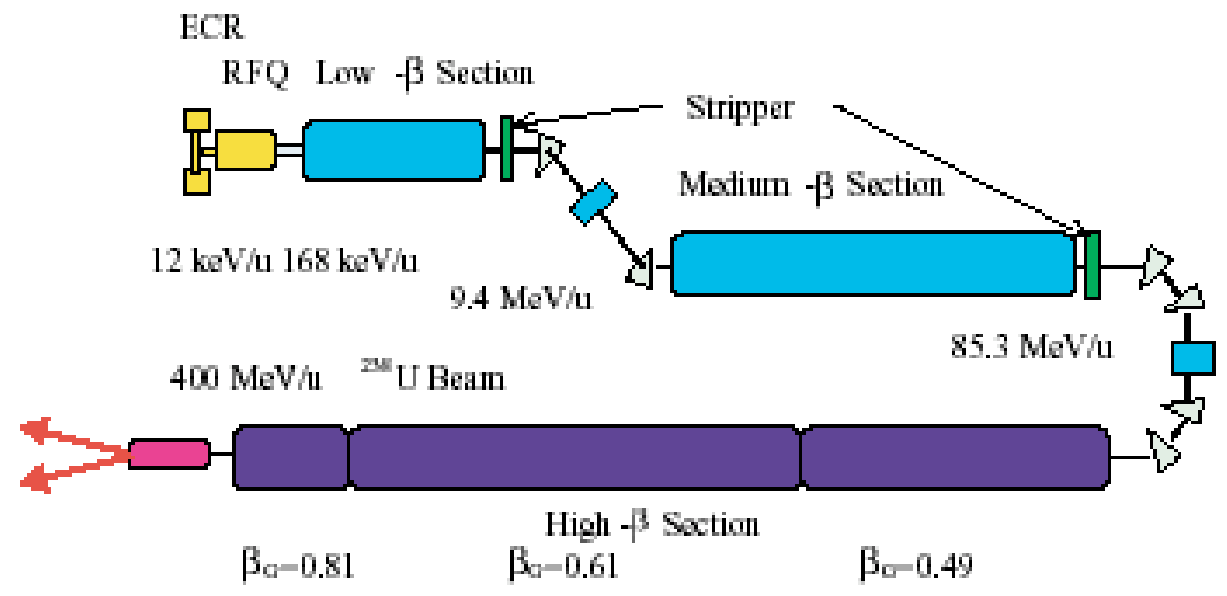

Figure 1. Elements of the proposed SCL from Ref. [1]

One characteristic of the SCL design, in order to achieve the required final beam intensity, is the simultaneous acceleration of multiple charge states. The expected full beam normalized emittance is $\varepsilon_{\mathrm{n}}=1.75 \pi \mathrm{mm}$-mrad, as it is shown in Figure 17 of Ref. [1]. The $100 \%$ beam bunch area at the end of the SCL is also estimated to be $\mathrm{S}=76 \pi$ $\mathrm{eV} / \mathrm{u}-\mu \mathrm{s}$, from Table VI of the same Ref. [1]. Both emittance and bunch area are quoted to include all the accepted charge states, and the multiple scattering effects in the two stripping targets. Table 1 of this report summarizes the main parameters of the SCL approach design. In particular we reproduce Tables V and VII of Ref. [1], respectively as Table 2 and Table 3, that report parameters of the High- $\beta$ Section of the SCL, and of the performance of both Stripping Targets. We shall take these parameters as the reference for our design based on the used of FFAG accelerators instead of SCL.

Table 1. Summary of the SCL Approach Design

\begin{tabular}{|l|l|l|l|}
\hline & \multicolumn{1}{|c|}{ Low- $\beta$} & \multicolumn{1}{c|}{ Medium- $\beta$} & \multicolumn{1}{c|}{ High- $\beta$} \\
\hline Aprox. Length, $\mathrm{m}$ & 55 & 150 & 200 \\
\hline Energy Range, $\mathrm{MeV} / \mathrm{u}$ & $0.168-9.4$ & $9.4-85.3$ & $85.3-404$ \\
\hline RF Frequency, $\mathrm{MHz}$ & $57.5-115$ & $172.5-345$ & 805 \\
\hline No. of Cavities & 85 & 195 & 136 \\
\hline No. Focusing Periods & -- & 58 & 38 \\
\hline
\end{tabular}

\section{Possible FFAG Scenarios}

We shall still impose the same performance requirements as in the original SCL proposal [1]. That is an average beam power of $400 \mathrm{kWatt}$ at the kinetic energy of 400 $\mathrm{MeV} / \mathrm{u}$ for ions of Uranium 238. As a consequence the average beam current on the Target is $4.2 \mu \mathrm{A}$-ion. Possible scenarios can make use of either one or two FFAG rings as shown schematically in Figure 2. 
Table 2. Basic Parameters of the High- $\beta$ Section of the SCL. From Ref [1]

TABLE V. Basic parameters of the high- $\beta$ linac section.

\begin{tabular}{|c|c|c|c|}
\hline Energy range $(\mathrm{MeV} / \mathrm{u})$ & $81-154$ & $154-326$ & $326-404$ \\
\hline Geometrical beta & 0.49 & 0.61 & 0.81 \\
\hline $\begin{array}{l}\text { Accelerating gradient } \\
(\mathrm{MV} / \mathrm{m})\end{array}$ & 10.54 & 13.01 & 15.99 \\
\hline $\begin{array}{l}\text { Cavity peak surface field } \\
(\mathrm{MV} / \mathrm{m})\end{array}$ & & 35.0 & \\
\hline Cavity length (cm) & 55 & 68 & 91 \\
\hline Cryomodule length (m) & 2.5 & 5.2 & 6.3 \\
\hline Number of cavities & 48 & 64 & 24 \\
\hline $\begin{array}{l}\text { Focusing lattice } \\
\text { rms fluctuation of rf }\end{array}$ & & FDO & \\
\hline field phase (deg) & & 0.3 & \\
\hline $\begin{array}{l}\text { ms fluctuations of the field } \\
\text { amplitude }(\%)\end{array}$ & & 0.3 & \\
\hline Number of focusing periods & 16 & 16 & 6 \\
\hline Length of quadrupole $(\mathrm{cm})$ & 25 & 41 & 41 \\
\hline $\begin{array}{l}\text { Length of the focusing } \\
\text { period }(\mathrm{m})\end{array}$ & 3.65 & 5.84 & 7.89 \\
\hline $\begin{array}{l}\text { Focusing gradient }(\mathrm{T} / \mathrm{m}) \\
\text { rms misalignments of the } \\
\text { ends of quadrupoles } \\
(\mathrm{mm})\end{array}$ & $12.0-20.0$ & $12.0-14.0$ & 15.0 \\
\hline
\end{tabular}

Table 3. Stripper Effect on the Uranium Beam. From Ref. [1]

TABLE VII. Stripper effect on the uranium beam.

\begin{tabular}{lcc}
\hline \hline Beam energy $(\mathrm{MeV} / \mathrm{u})$ & 9.43 & 85.2 \\
Medium & Liquid lithium film & Rotating carbon wheel \\
Thickness $\left(\mathrm{mg} / \mathrm{cm}^{2}\right)$ & 0.1 & 15 \\
Energy loss $(\mathrm{MeV} / \mathrm{u})$ & 0.01 & 4.0 \\
Mean charge state & 71 & 89 \\
Charge states & $69-73$ & $88-91$ \\
Beam fraction within design charge states $(\%)$ & 69 & 96 \\
ms angle of transverse beam scattering (mrad) & 0.2 & 0.44 \\
ms energy spread $(\mathrm{keV} / \mathrm{u})$ & 4.1 & 13.5 \\
\hline \hline
\end{tabular}

The Scenario with a single FFAG ring (Figure 2 above) makes use of the first half of the SCL design up to the energy of $80 \mathrm{MeV} / \mathrm{u}$. This includes the FE, the Low- $\beta$ and the Medium- $\beta$ Sections and both stripping targets. The High- $\beta$ Section is entirely replaced by one FFAG ring that accelerates the beam from 80 to $400 \mathrm{MeV} / \mathrm{u}$ at the reference charge state $\mathrm{Q}=90$. The FFAG ring has a circumference of $204 \mathrm{~m}$ that is about the length of the High- $\beta$ Section. The Scenario may offer some cost advantages when more conventional technology is traded with superconducting RF technology. 

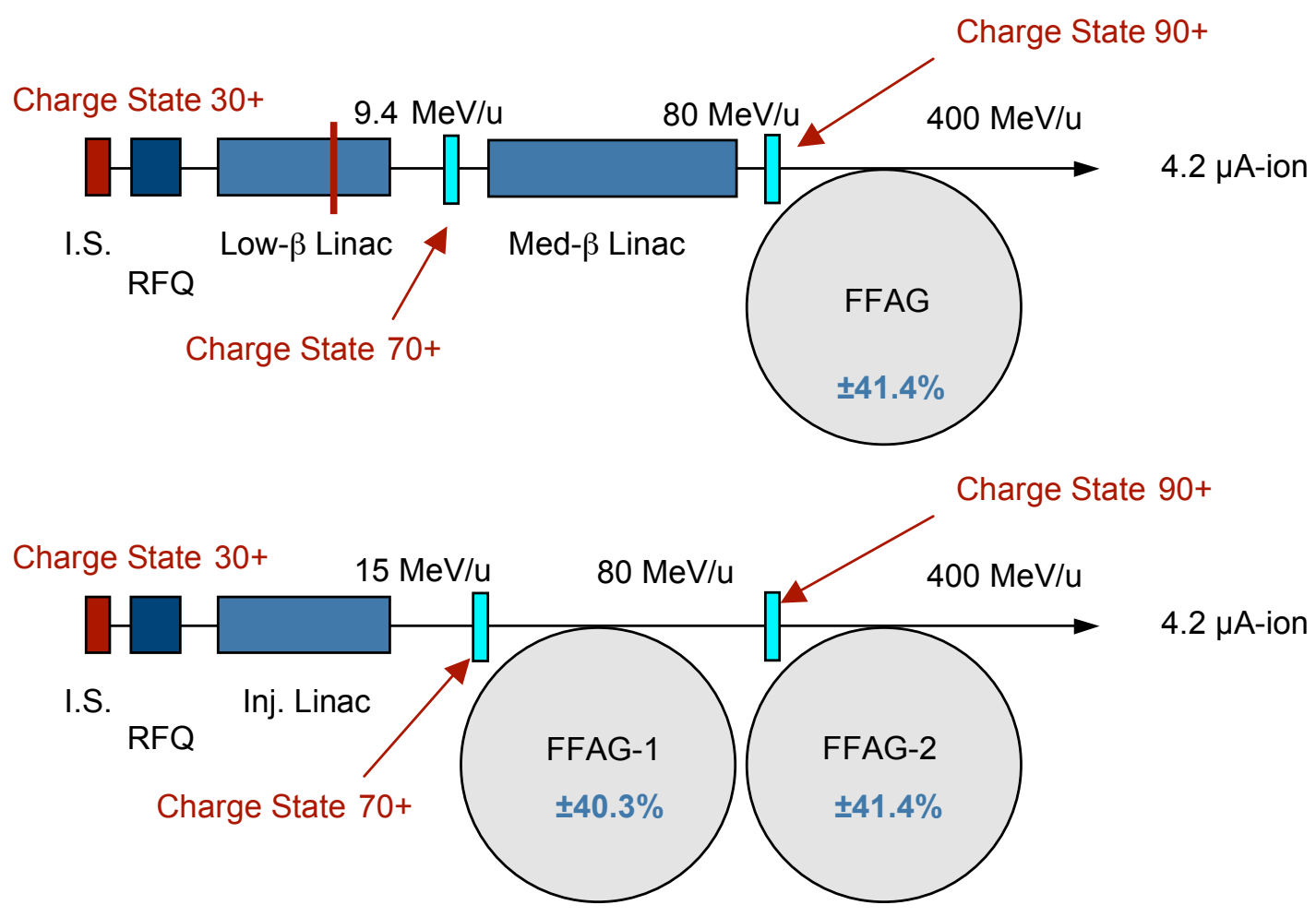

Figure 2. FFAG-based accelerator for RI and ENF production

Figure above. Scenario with a single FFAG Ring

Figure below. Scenario with two FFAG Rings

In the two FFAG ring scenarios (Figure 2 below) an extra ring is added for acceleration between 15 and $80 \mathrm{MeV} / \mathrm{u}$. The two rings have the same circumference of $204 \mathrm{~m}$; they can be located in the same tunnel enclosure either on top of each other, or concentric to each other at close radial distance. The injection and extraction energies of each ring have been determined by setting a momentum range for acceleration around \pm $40 \%$. In this scenario the FE and the Low- $\beta$ Section of the SCL are retained as designed, but an extra section is added for acceleration from 9.4 to $15 \mathrm{MeV} / \mathrm{u}$. This section can be made of cavities operating at $172.5 \mathrm{MHz}$ similar to those of the Medium- $\beta$ Section of the SCL.

To get the required average beam intensity, as in the SCL approach, also the two FFAF scenarios are to be capable to accelerate multiple charge states, around $\mathrm{Q}=70$ in FG-1 and Q $=90$ in FFAG-2. Nevertheless in our estimates we shall assume an average charge state in each ring as specified.

Intentional beam losses are also expected: (1) At the FE the beam will have to be pre-chopped and pre-bunched for more efficient acceleration in the FFAG rings where one wants to avoid large losses at higher energies. Overall we may expect a beam transmission from FE to rings between 50 and $70 \%$. (2) Another location where we may expect appreciable beam losses is at the stripping targets. Table 3 shows that the beam loss is about $30 \%$ at the first stripping target, and $4 \%$ at the second. The scenario with 2 
FFAG rings has the advantage of raising the energy of the injection Linac to $15 \mathrm{MeV} / \mathrm{u}$. at this larger energy we could indeed expect a better beam survival for some charge state distribution. (3) There are also controlled beam losses at the location of collimators, around the injection and extraction systems, to protect the magnet components from active radiation. These losses could range to around $10 \%$. In sum, in order to get the required average beam of $4.2 \mu \mathrm{A}$-ion on the main Target, the $\mathrm{FE}$ ought to deliver an equivalent beam average current of about $10 \mu \mathrm{A}$-ion.

\section{Design of the FFAG Rings}

The two FFAG rings have the same circumference and the same identical lattice structure that is summarized in Table 4. Each of them is made of an unbroken sequence of FDF triplets as shown in Figure 3. We are adopting the NSL choice that yields a more compact magnet configuration and considerably stronger focusing. There are two major drifts: a long one, $s$, and a minor one, $g$, that separate the magnets. These are sector magnets with parallel entrance and exit faces. The field profile is linear, namely an superposition of a dipole and a quadrupole (shifted) field. The reference orbit is taken to be at injection. The beam is injected on a inner orbit and, as it is accelerated, the trajectory spirals toward to the outside, and it is extracted from an outer orbit. The good feature of the FFAG ring is that bending and focusing fields are kept constant during acceleration, allowing thus a higher acceleration rate.

With the same circumference of $204 \mathrm{~m}$ we have considered different periodicity $\mathrm{N}$ $=80,60$ and 40, where a period is made of a FDF triplet as shown in Figure 3. A lower periodicity makes the lattice unstable at the high-energy end, and a higher periodicity is unpractical. Table 4 gives the summary of the lattice geometry. Two main parameters are the length $s$ of the long drift that one desires long enough to accommodate RF cavities, collimators, and injection and extraction components, and the radial width $w$ required for the momentum excursion during acceleration. The width $w$ is to be small enough to match the radial extension of the RF cavities especially in the case of very high frequency.

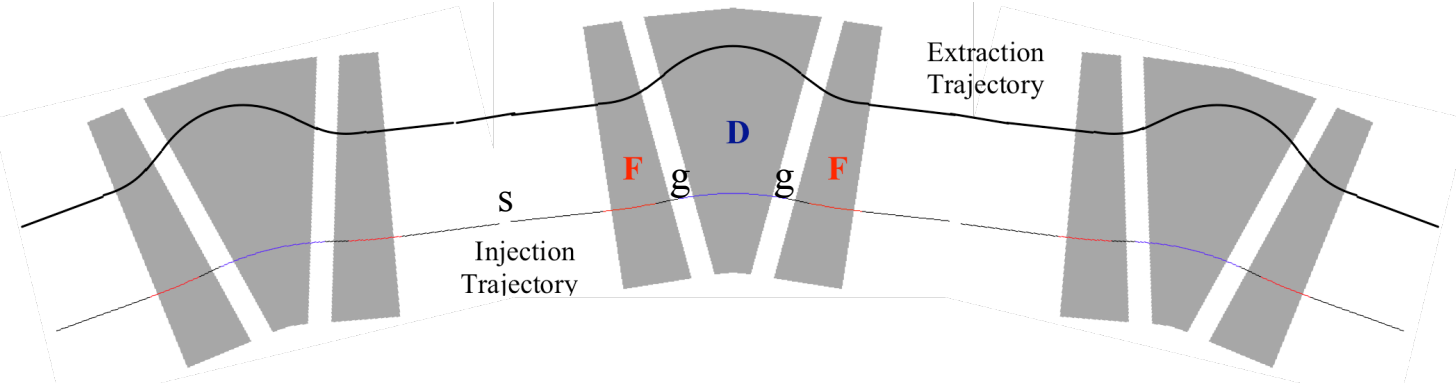

Figure 3. The FDF triplet period of the FFAG rings

Figure 4 is the plot of the lattice functions along the length of one period. Because of the adoption of the NSL, the lattice functions vary with energy as it is shown by the variation of the betatron tunes during acceleration in Figure 5. To the left of the same Figure, closed orbits are plotted at different momenta in the acceleration cycle along the length of one period. From these plots we can infer the required radial excursion $w$. 
Figure 6 gives the magnetic field profile in the two sector magnets for both FFAG rings. All Figures 4 to 6 refer to periodicity $\mathrm{N}=80$. If $\mathrm{C}$ denotes the circumference, the period length $\mathrm{L}=\mathrm{C} / \mathrm{N}$. The $\beta_{\mathrm{H}, \mathrm{V}}$ lattice functions scale linearly with $\mathrm{L}$, and the dispersion function $\eta$ with the square of $\mathrm{L}$. The betatron tunes $\mathrm{Q}_{\mathrm{H}}$ and $\mathrm{Q}_{\mathrm{V}}$ increase linearly with $\mathrm{N}$ since the phase advance per period is kept to be the same in all cases, independently of the periodicity $N$. The off-momentum variable $\delta=\left(p-p_{\text {inj }}\right) / p_{\text {inj }}$ is used as the abscissa of Figure 5, where $\mathrm{p}$ is the actutal particle momentum and $\mathrm{p}_{\text {inj }}$ the value at injection. At extraction $\delta_{\text {ext }}=\left(p-p_{\text {inj }}\right) / p_{\text {inj }}=1.35$ corresponding to a relative momentum range $\Delta= \pm$ $40-41 \%$ in both FFAG rings.

Table 4. Structure of the FFAG Rings: identical to both Rings

Circumference

Number of Periods

Period Length, $\mathrm{m}$

Long Drift $s, \mathrm{~m}$

Short Drift $g, \mathrm{~m}$

F-Sector Arc Length, m

D-Sector Arc Length, $m$

Radial Width $w, \mathrm{~cm}$

Packing Factor, \%
$204 \mathrm{~m}$

80

2.55

1.089

0.129

0.301

0.602

11.2

47.2
60

3.40

1.452

0.172

0.401

0.802

18.8

47.2
40

5.10

2.178

0.258

0.602

1.203

37.1

47.2
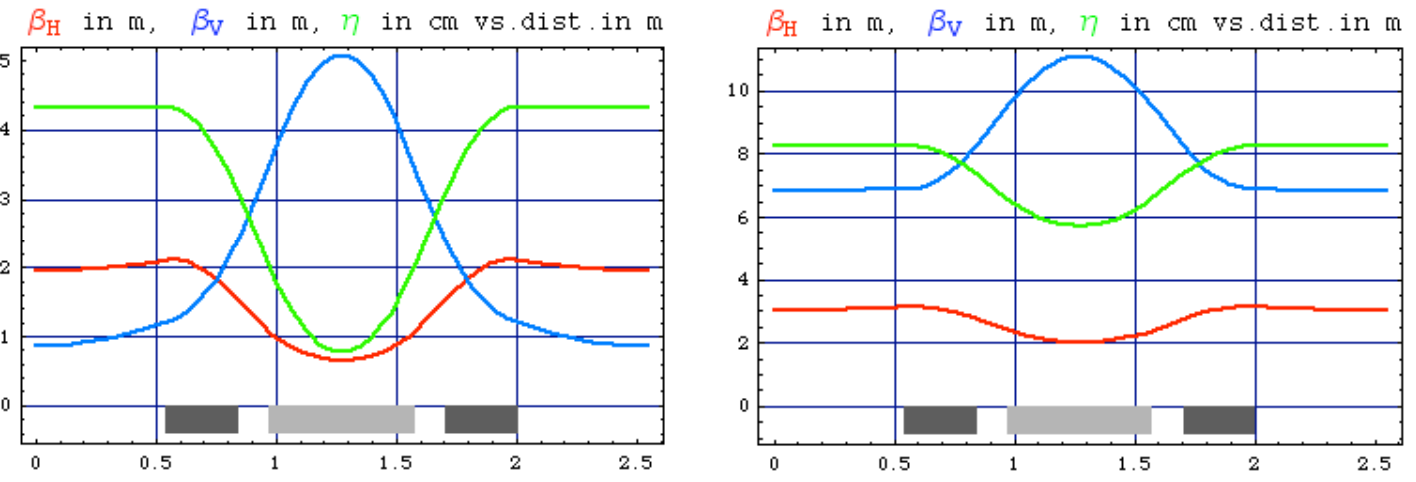

Figure 4. Lattice Functions along the length of a Period $(\mathrm{N}=80)$
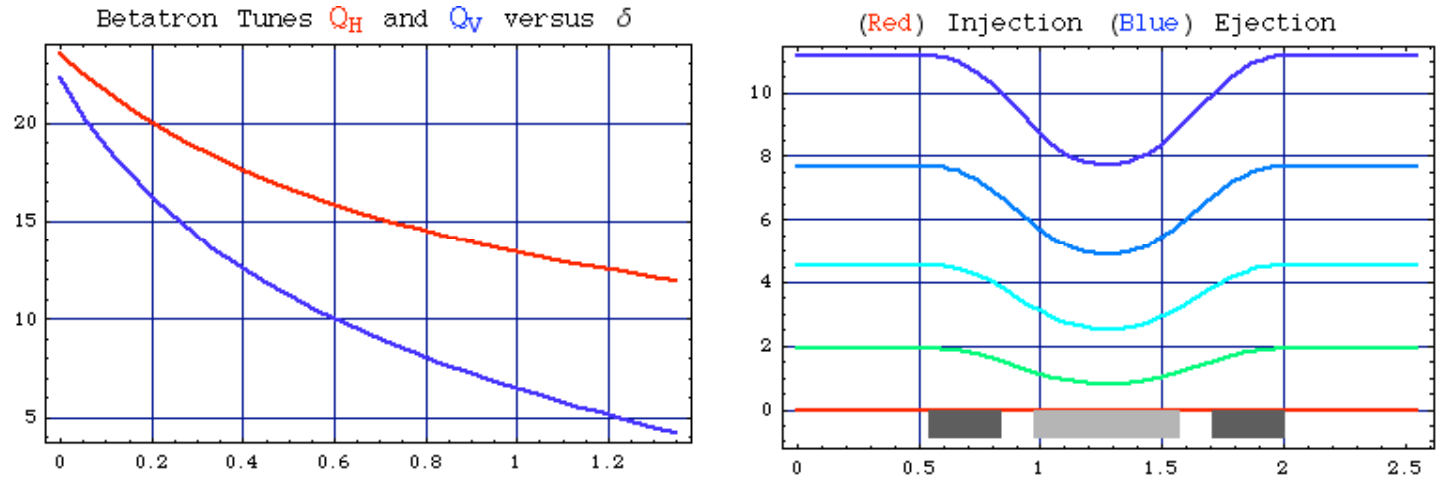

Figure 5. Betatron Tunes and Closed Orbits during the acceleration cycle $(\mathrm{N}=80)$ 

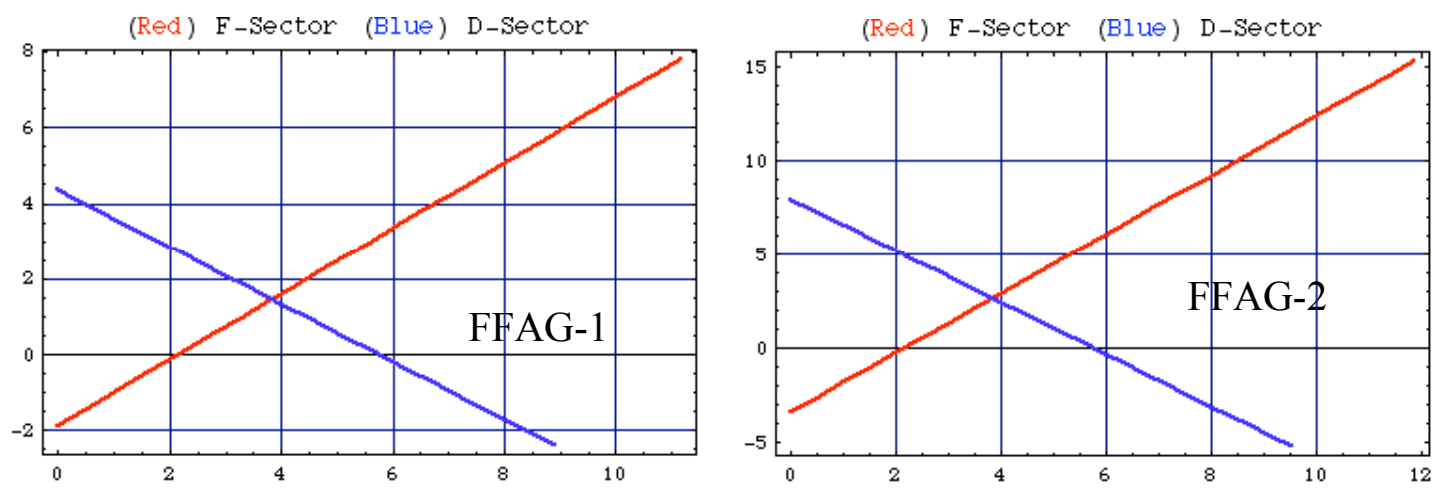

Figure 6. Field Profiles $(\mathrm{kG})$ in the two FFAG rings vs. radial location $\mathrm{x}(\mathrm{cm})(\mathrm{N}=80)$

The required strength of the magnets is shown in Figure 6 for $\mathrm{N}=80$ periodicity. The field strength at injection remains unchanged, but it is considerably lower by decreasing the periodicity N. For instance the maximum field in the F-sector magnet at extraction in the FFAG-2 ring which is the most demanding drops from $15.3 \mathrm{kGauss}$ for $\mathrm{N}=80$ down to $14.2 \mathrm{kGauss}$ for $\mathrm{N}=60$ and $12.1 \mathrm{kGauss}$ for $\mathrm{N}=40$.

\section{Possible Acceleration Schemes}

The average beam power $\mathrm{P}=400 \mathrm{kWatt}$ at the kinetic energy $\mathrm{E}=400 \mathrm{MeV} / \mathrm{u}$ is obtained with an output average beam current $\mathrm{I}=\mathrm{P} / \mathrm{AE}=4.2 \mu \mathrm{A}$-ions, where $\mathrm{A}=238$ is the mass number of ions of Uranium at the (average) charge state $Q=+90$. As an example, if the FFAG accelerator complex operates at the repetition rate $f_{\text {rep }}=1,000$ beam pulses for second $(1 \mathrm{kHz})$ on the external Target, the total number of ions required per acceleration cycle is $\mathrm{N}_{\text {ions }}=\mathrm{I} / \mathrm{ef}_{\text {rep }}=2.63 \times 10^{10} /$ pulse, and e is the electron charge. At the repetition rate of $10 \mathrm{kHz}$ the required number of ions is ten times smaller, that is $2.63 \times 10^{9} /$ pulse. The main issue is how to stack such large number of ions in the first FFAG ring considering the limitation in current, emittance, and time duration of the ion source.

For each of the two FFAG scenarios, with a single or two FFAG rings, we have considered three cases:

A. Acceleration at the repetition rate of $1 \mathrm{kHz}$ with constant frequency, broadband cavity system.

B. Acceleration at the repetition rate of $10 \mathrm{kHz}$ with the Harmonic Number Jump (HNJ) method.

C. $\quad$ CW mode of operation with HNJ.

\section{Acceleration with Broadband Cavities at $1 \mathrm{kHz}$}

Table 5 summarizes the main parameters of our design assuming a repetition rate $\mathrm{f}_{\text {rep }}=1 \mathrm{kHz}$. We have assumed acceleration with a broadband, fixed-frequency RF cavity system as developed at KEK in Japan employing Metal Alloy (MA). Because of the high repetition rate required, acceleration with ferrite driven cavities does not seem feasible at 
cause of the limitation to about few $\mathrm{MHz} / \mathrm{ms}$ frequency sweep that can at most be achieved.

Table 5. Main Beam Parameters of the FFAG-based Accelerator. Repetition Rate $=1 \mathrm{kHz}$. Circumference $=204 \mathrm{~m} . \mathrm{N}=2.63 \times 10^{10} /$ pulse.

\begin{tabular}{|c|c|c|c|c|c|}
\hline & & \multicolumn{2}{|c|}{ FFAG-1 (+70) } & \multicolumn{2}{|c|}{ FFAG-2 (+90) } \\
\hline & & Injection & Extraction & Injection & Extraction \\
\hline Kinetic Energy & $\mathrm{MeV} / \mathrm{u}$ & 15 & 80 & 80 & 400 \\
\hline$\beta$ & & 0.1767 & 0.3885 & 0.3885 & 0.7131 \\
\hline$\beta \gamma$ & & 0.1795 & 0.4217 & 0.4217 & 1.0170 \\
\hline Revol. Freq. & $\mathrm{MHz}$ & 0.2597 & 0.5697 & 0.5710 & 1.0454 \\
\hline Revol. Period & $\mu \mathrm{s}$ & 3.851 & 1.755 & 1.751 & 0.957 \\
\hline Emitt. full norm. & $\pi \mathrm{mm} \mathrm{mrad}$ & \multicolumn{2}{|c|}{100} & \multicolumn{2}{|c|}{100} \\
\hline Bunch Area, rms & $\mathrm{eV} / \mathrm{u}-\mathrm{s}$ & \multicolumn{2}{|c|}{0.02} & \multicolumn{2}{|c|}{0.02} \\
\hline Space-Charge $\Delta v$ & & 0.020 & 0.0085 & 0.018 & 0.0069 \\
\hline Harmon. No. (*) & & \multicolumn{2}{|c|}{6} & \multicolumn{2}{|c|}{6} \\
\hline RF Frequency & $\mathrm{MHz}$ & 1.558 & 3.418 & 3.426 & 6.273 \\
\hline RF peak Voltage & MVolt & \multicolumn{2}{|c|}{0.8} & \multicolumn{2}{|c|}{1.6} \\
\hline RF Phase & & \multicolumn{2}{|c|}{$60^{\circ}$} & \multicolumn{2}{|c|}{$60^{\circ}$} \\
\hline Accel. Period & $\mathrm{ms}$ & \multicolumn{2}{|c|}{0.758} & \multicolumn{2}{|c|}{0.726} \\
\hline No. of Revol. & & \multicolumn{2}{|c|}{319} & \multicolumn{2}{|c|}{611} \\
\hline Ave. Beam Curr. & mA-ion & 1.093 & 2.397 & 2.403 & 4.400 \\
\hline RF Beam Power & kWatt & 53.0 & 116. & 300. & 549. \\
\hline $\begin{array}{l}\text { No. of Cavities } \\
\begin{array}{r}\mathrm{N}=80 \text { periods } \\
\mathrm{N}=40 \text { periods }\end{array}\end{array}$ & & \multicolumn{2}{|c|}{$\begin{array}{c}40 \\
14-20\end{array}$} & \multicolumn{2}{|c|}{$\begin{array}{c}80 \\
28-40\end{array}$} \\
\hline RF Cavity Power & MWatt & \multicolumn{2}{|c|}{4.0} & \multicolumn{2}{|c|}{8.0} \\
\hline
\end{tabular}

(*) Also number of beam bunches.

A Finemet cavity is typically made with 3 gaps for a total length of about $60 \mathrm{~cm}$, and a peak voltage of $20 \mathrm{kVolt}$ in the middle of the frequency range shown in Table 5. There is nevertheless a significant drop of voltage $(\sim 30 \%)$ on both sides of the bandwidth that may need to be compensated with either adjusted phase program or by increasing the number of cavities. The actual accelerating filed achieved in the Finemets cavity is close to $100 \mathrm{lkVolt} / \mathrm{m}$, but this value applies to the active region of the core. When the cavity is packaged in a multi-layer pancake the actual accelerating gradient is diluted [11].

If we take the parameters of Table 5, one long straight section $(s)$, about $1 \mathrm{~m}$ in length for $\mathrm{N}=80$ periodicity, can accommodate a multi-gap cavity for a total of $20 \mathrm{kVolt}$ peak. Thus one needs 40 broadband cavities for a total of 0.8 MVolt in the FFAG-1 ring, and 80 broadband cavities for a total of $1.6 \mathrm{MVolt}$ in the FFAG-2 ring. With a periodicity $\mathrm{N}=40$ the straight section $(s)$ is twice as long $(\sim 2 \mathrm{~m})$ and two or three cavities may be locally installed for a more efficient occupancy. In both cases the cavities are demanding in power as they dissipate more than $100 \mathrm{kWatt}$ in a 3-gap configuration [11]. The total 
dissipated power is thus in several MWatt range, considerably higher that the actual beam RF power of only a fraction of MWatt.

\section{Injection Issues at $1 \mathrm{kHz}$ Repetition Rate}

Inspection of Table 5 shows that the beam circulating current at injection into FFAG-1 is $1.1 \mathrm{~mA}$-ion, and $2.4 \mathrm{~mA}$-ion into FFAG-2. This is the main issue (and maybe problem) of this design. How can we stack such large current in the ring from a limited ion source? We obviously need some type of multi-turn injection. Charge exchange method is to be ruled out. One has thus to rely on the old fashion betatron stacking.

The beam is bunched at the FE at $57.5 \mathrm{MHz}$ and enters either of the two FFAGs at that frequency. The beam is also pre-chopped by $70 \%$ at the RF frequency at injection to control beam losses during the RF capture that could activate the ring components. There are thus 26 bunches in each of the 6 RF standing-by RF buckets at injection into FFAG-1, or 12 bunches in each of the corresponding RF buckets at injection into the high-energy FFAG in the single ring scenario.

The minimum required betatron acceptance of the $\operatorname{ring} A=\varepsilon_{\mathrm{n}} /(\beta \gamma)_{\text {inj }}$ where $\varepsilon_{\mathrm{n}}$ is the full beam normalized emittance shown in Table 5. Even in the case betatron stacking is entirely done in the horizontal plane, in the worst scenario, because of coupling, both horizontal and vertical emittance will equal each other. Let $n$ also be the number of injected turns, then the following relation holds

$$
A=n \varepsilon_{\mathrm{sn}} / \beta \gamma \eta
$$

with $\eta$ the betatron stacking efficiency, and $\varepsilon_{\mathrm{sn}}$ the normalized full emittance of a single turn from the FE. Operation with ions of gold in the AGS Booster [12] has demonstrated that it is possible to stack $n=40$ turns with an efficiency $\eta=40 \%$. The maximum value of the $\beta_{\mathrm{V}}$ lattice function in the ring with $\mathrm{N}=80$ periodicity is $5.1 \mathrm{~m}$. Table 6 gives the results of the ion source requirements and ring physical acceptance.

Table 6. Betatron Stacking with $n=40$ and $\eta=40 \%$ at $\mathrm{f}_{\text {rep }}=1 \mathrm{kHz}$

FFAG-1 $\quad$ FFAG-2

$\begin{array}{lll}A & 560 \pi \mathrm{mm}-\mathrm{mrad} & 240 \pi \mathrm{mm}-\mathrm{mrad} \\ \varepsilon_{\mathrm{sn}} / \beta \gamma & 5.6 \pi \mathrm{mm}-\mathrm{mrad} & 2.4 \pi \mathrm{mm}-\mathrm{mrad} \\ \mathrm{I}_{\mathrm{s}} & 28 \mu \mathrm{A}-\mathrm{ion} & 60 \mu \mathrm{A}-\mathrm{ion} \\ 3 \mathrm{~b}=3\left(A \beta_{\mathrm{V}}\right)^{1 / 2} & 165 \mathrm{~mm} & 105 \mathrm{~mm} \\ \mathrm{~T}_{\mathrm{s}} & 154 \mu \mathrm{s} & 70 \mu \mathrm{s}\end{array}$

$I_{s}$ is the single turn current obtained by dividing the circulating current $t$ injection shown in Table 5 by the number of injected turns $n$. we have allowed a $50 \%$ clearance on top of the full beam height in the estimate of the required vertical magnet gap $3 \mathrm{~b}$. The required pulse length $T_{\mathrm{s}}$ from the ion source, also shown in Table 6 , is given as the product of $n$ with the revolution period. 
It is not easy to achieve these parameters from the ECR source. The addition of an Accumulator Ring sitting in front of the two FFAG accelerator rings does not ameliorate the situation because it would just transfer the betatron stacking problem with the same ion source requirements to that ring. An alternative is to find a way somehow to transfer the beam from the ECR in a following EBIS structure where it can be stored, and eventually continued ionized, by the electron beam. Then once enough ions have been stored, the beam is extracted from the EBIS in a single pulse and transported through the RFQ and the Linac prior to injection into either one of the FFAG rings. The FE layout is then changed as shown in Figure 7. The ECR-EBIS-RFQ combination operates at $1 \mathrm{kHz}$ repetition rate. Thus over a period of one millisecond the beam is continuously injected from the ECR and stored into the EBIS. At the end of that period of time $2.63 \times 10^{10}$ ions have been accumulated preserving their original charge states $Q=28-29$. The pulse is extracted with a duration of $3.5 \mu \mathrm{s}$ (or $1.5 \mu \mathrm{s}$ for the single high-energy FFAG scenario) to allow also a gap for the injection kicker fall-off time. Correspondingly, the instantaneous beam current in exit from the EBIS is $1.1 \mathrm{~mA}$-ion (or $2.4 \mathrm{~mA}$-ion). The full beam normalized emittance should not exceed $100 \pi \mathrm{mm}-\mathrm{mrad}$ to limit the height of the magnet gap to the value given in Table 6 , and not be less than $10 \pi \mathrm{mm}$-mrad to avoid a space-charge tune depression exceeding 0.2. Transfer of the beam from the ECR source to the EBIS and storage in EBIS need of course to be demonstrated within the beam parameters range just specified.

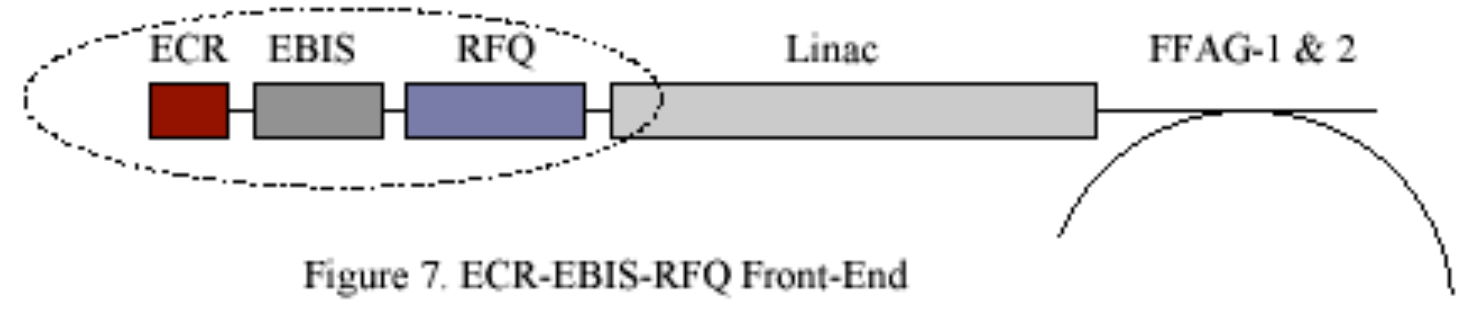

\section{Acceleration by HNJ}

A higher repetition rate is desirable to ease on the performance requirements of the ions source and of multi-turn injection into FFAG-1. At the repetition rate frep $=10$ $\mathrm{kHz}$ the required number of ions is $\mathrm{N}_{\text {ions }}=2.63 \times 10^{9}$ per pulse and the acceleration period should not exceed $100 \mu \mathrm{s}$. For a higher repetition rate we should consider a different RF system for acceleration. That is the method of Harmonic Number Jump (HNJ) [9]. This method allows the use of superconducting RF cavities at very high constant frequency, in the range of several hundreds $\mathrm{MHz}$ or even in the $\mathrm{GHz}$ range. Acceleration requires a programmed energy gain that varies between cavity crossings to allow the change of the transit time between cavities that corresponds to the jump of one or more RF harmonics. If $f_{R F}$ is the RF frequency, obviously the relation $f_{R F}=h \beta c / C$ holds where $\mathrm{h}$ is the (local, that is between two consecutive cavity crossings) harmonic number, $\mathrm{C}$ the distance between cavities, and $\beta \mathrm{c}$ the beam velocity. In a synchrotron the harmonic number $\mathrm{h}$ is kept constant; as the beam velocity $\beta \mathrm{c}$ varies, then the $\mathrm{RF}$ frequency $f_{R F}$ is adjusted accordingly. The HNJ method, on the other end, requires that $f_{\mathrm{RF}}$ is kept constant so that as the beam velocity $\beta \mathrm{c}$ changes the harmonic number $\mathrm{h}$ will 
have to vary accordingly. This can be achieved only with a proper program of energy gain between cavity crossings [9]. It is to be pointed out that, since the harmonic number $\mathrm{h}$ reduces during acceleration, the number of beam bunches at injection into the first ring cannot be larger than then harmonic number at extraction from the second ring. That is the beam injected into the first ring has a duration that is a fraction of the revolution period at the same time given by the ratio $\beta_{2} / \beta_{1}=4.04$ of the final to the initial value of the beam velocity. Consequently the average current of the injected beam pulse is larger by the same factor of the average value over the whole circumference. In the case of a single high-energy FFAG ring the ratio $\beta_{2} / \beta_{1}=1.84$.

The FFAG ring has $M$ equally spaced localized groups of cavities each with $\mathrm{N}_{c}$ cavities, described later, as shown in Figure 8. Each group of cavities applies an energy gain that is a function of the beam energy at the moment of the $n$-th crossing. The variation of $h$ with $\beta$ can be calculated precisely on a computer, but here we use a linear approximation (a very good one indeed!)

$$
\begin{aligned}
\Delta \mathrm{E}_{\mathrm{n}+1} & =\mathrm{E}_{0} \beta_{\mathrm{n}}{ }^{2} \gamma_{\mathrm{n}}{ }^{3} \Delta \mathrm{h} /\left(1-\alpha_{\mathrm{p}} \gamma_{\mathrm{n}}{ }^{2}\right) \mathrm{h}_{\mathrm{n}} \\
& =(\mathrm{Q} / \mathrm{A}) \mathrm{eV}_{\mathrm{n}} \sin \phi_{\mathrm{RF}}
\end{aligned}
$$

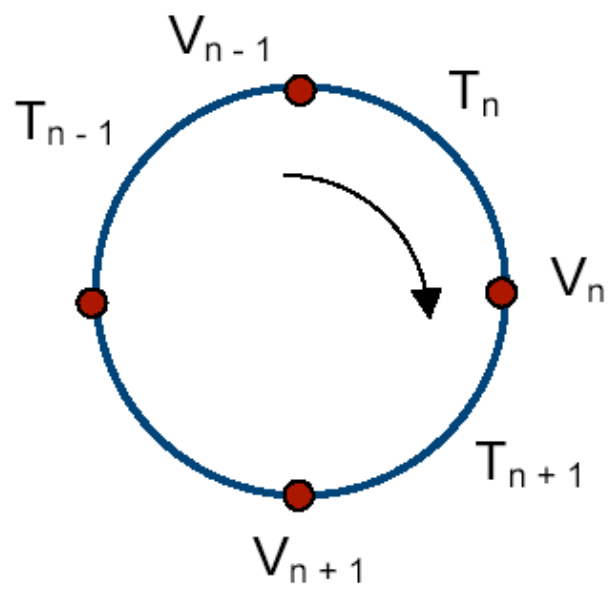

Figure 8. Multi-Cavity FFAG Layout

where $\mathrm{E}_{0}$ is the ion rest energy, $\beta_{\mathrm{n}}$ and $\gamma_{\mathrm{n}}$ the velocity and energy relativistic factors at the $\mathrm{n}$-th crossing, $\alpha_{\mathrm{p}}$ the momentum compaction that may also vary with the beam energy, $\mathrm{h}_{\mathrm{n}}$ the harmonic number between the (n-1)-th and the $\mathrm{n}$-th crossing, and $\phi_{\mathrm{RF}}$ the constant $\mathrm{RF}$ phase. Finally $\Delta h=h_{n+1}-h_{n}<0$ is the harmonic number jump.

Table 8 gives the beam and RF parameters for the case the HNJ method is applied to both FFAG rings with a circumference $\mathrm{C}=204 \mathrm{~m}$. The repetition rate is $10 \mathrm{kHz}$ in which case the number of ions accelerated is $2.63 \times 10^{9} /$ pulse. The adopted harmonic number jump is $\Delta \mathrm{h}=-1$. All the other beam parameters remain as specified in Table 5 .

The advantage of the higher repetition rate is that the time the beam spends in the ring is very short and circulates only for very few revolutions. This reduces considerably our concern of the effects on the beam stability and loss of the multiple-resonce crossing with the NSL.

We found convenient to adopt a RF of $805 \mathrm{MHz}$. The internal dimension of the cavity should then not exceed $20 \mathrm{~cm}$. The width $w$ reported in Table 4 is significant in this regard and seems to exclude the case of low periodicity $\mathrm{N}=40$. 
Table 8. Beam and RF Parameters for $10 \mathrm{kHz}$ Repetition Rate with HNJ

\begin{tabular}{|c|c|c|c|c|}
\hline & \multicolumn{2}{|c|}{ FFAG-1 } & \multicolumn{2}{|c|}{ FFAG-2 } \\
\hline & Injection & Extraction & Injection & Extraction \\
\hline $\begin{array}{l}\text { Pulse Length } \\
\text { Bunching Freq. } \\
\text { No. of Bunches }\end{array}$ & \multicolumn{4}{|c|}{$\begin{array}{c}0.957 \mu \mathrm{s} \\
57.5 \mathrm{MHz} \\
55 \\
\end{array}$} \\
\hline Bunch Area & \multicolumn{4}{|c|}{$10 \mu \mathrm{eV} / \mathrm{u}-\mathrm{s} \mathrm{rms}$} \\
\hline Emittance & \multicolumn{4}{|c|}{$25 \pi \mathrm{mm}$-mrad full normalized ( $\mathrm{Dn}=0.2 \mathrm{sp} . \mathrm{ch})}$. \\
\hline$f_{R F}$ & \multicolumn{2}{|c|}{$806.03 \mathrm{MHz}$} & \multicolumn{2}{|c|}{$803.93 \mathrm{MHz}$} \\
\hline Rev. Frequency & $0.2597 \mathrm{MHz}$ & $0.5697 \mathrm{MHz}$ & $0.5710 \mathrm{MHz}$ & $1.0454 \mathrm{MHz}$ \\
\hline $\mathrm{h}($ total) & $8 \times 388$ & $8 \times 176$ & $4 \times 352$ & $4 \times 192$ \\
\hline Ave. Current & $109 \mu \mathrm{A}$-ions & $241 \mu \mathrm{A}-$-ions & $240 \mu \mathrm{A}$-ions & $440 \mu \mathrm{A}-$-ions \\
\hline No. Cavities & \multicolumn{2}{|c|}{8 groups -2 cavities each } & \multicolumn{2}{|c|}{4 groups -4 cavities each } \\
\hline RF Phase & \multicolumn{2}{|c|}{$30^{\circ}$} & \multicolumn{2}{|c|}{$60^{\circ}$} \\
\hline pk RF Voltage & \multicolumn{2}{|c|}{$2 \mathrm{MV} /$ Cavity } & \multicolumn{2}{|c|}{$8 \mathrm{MV} /$ Cavity } \\
\hline Acc. Period & \multicolumn{2}{|c|}{$74.0 \mu \mathrm{s}$} & \multicolumn{2}{|c|}{$54.0 \mu \mathrm{s}$} \\
\hline no. Revolutions & \multicolumn{2}{|c|}{$26+4 / 8$} & \multicolumn{2}{|c|}{40} \\
\hline RF Power & $8.32 \mathrm{~kW} /$ cavity & 109. kW /cavity & $54.1 \mathrm{~kW} /$ cavity & 758. kW/cavity \\
\hline
\end{tabular}

\section{Superconducting RF Cavities}

The required energy gain profile (total peak RF voltage per group of cavities) across the radial width is plotted in Figure 9. The voltage profile is about linear with the radial displacement of the beam during acceleration. Cavities are obviously superconducting, all operating very close to $805 \mathrm{MHz}$, taken from the experience with the development of the same type of cavities for the Spallation Neutron Source [10]. Cavities have a single cell and are all tuned independently from each other. The gap is related to the RF wavelength by the relation $g=\lambda_{R F} \beta_{0} / 2$ where $\beta_{0}$ is the nominal value of velocity kept constant and the same for all the cavities in the same ring. During the n-th cavity crossing the peak total voltage $V_{n}$ is related to the surface axial field $\xi_{n}$ through the relation $V_{\mathrm{n}}=\mathrm{g} \xi_{\mathrm{n}} \operatorname{TTF}\left(\beta_{0} / \beta_{\mathrm{n}}\right)$ where TTF is the Transit Time Factor that varies with the beam velocity $\beta_{\mathrm{n}}$. Te surface axial field $\xi_{\mathrm{n}}$ is plotted in Figure 10.
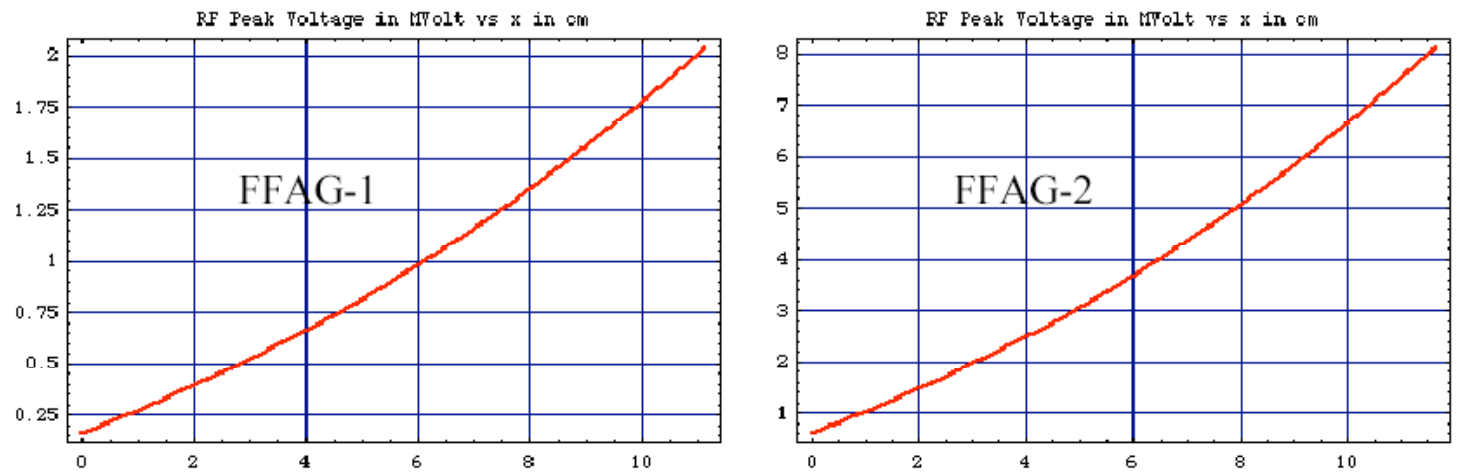

Figure 9. RF Voltage Profile in Mvolt vs. Radial Displacement x in cm 

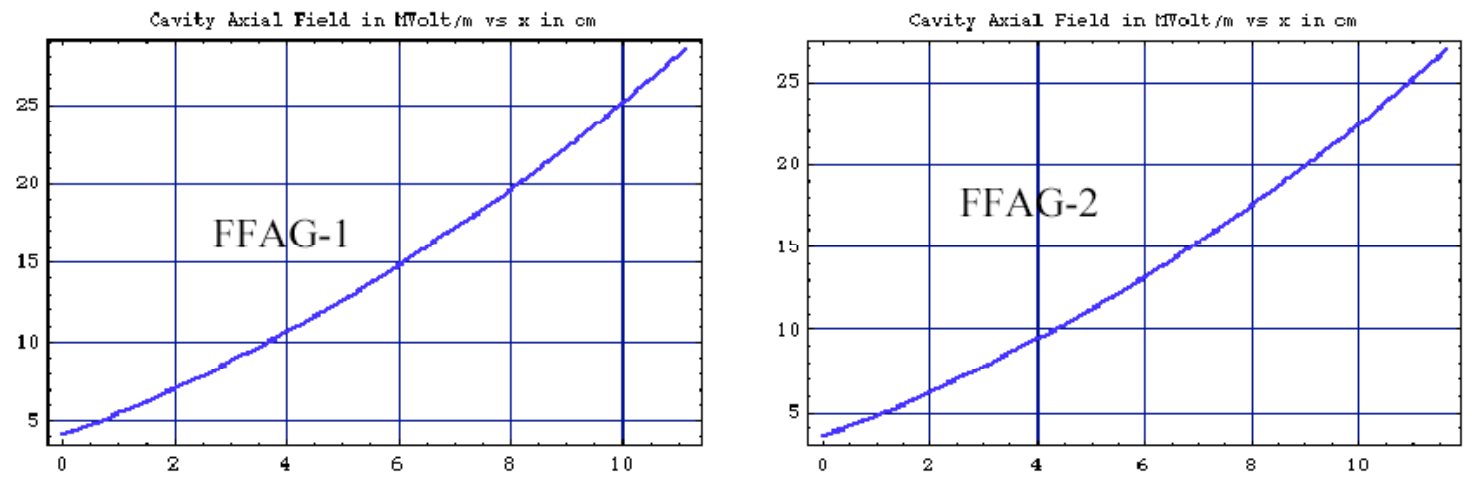

Figure 10. Total Surface Axial Field for each RF Cavity

The surface axial field is about the same for the cavities of both rings. It can be obtained with three cavities located next to each other, as shown in Figure 11, one operating in a $\mathrm{TM}_{01}$ mode at the constant field $\xi=16 \mathrm{MVolt} / \mathrm{m}$ corresponding to the middle value of the curves of Figure 10, and the other two in $\mathrm{TM}_{11}$ mode with a linear field $\xi= \pm 6 \mathrm{MVolt} / \mathrm{m}$ corresponding to the slope of the curves also shown in Figure 10.

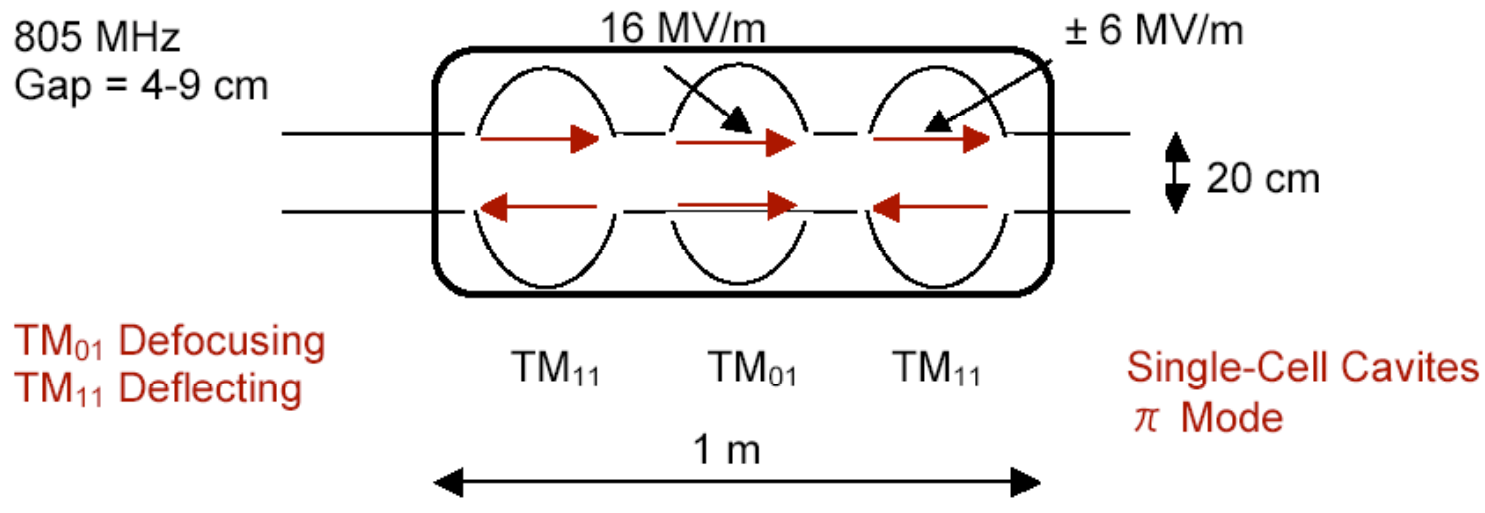

Figure 11. Combination of 3 cavities to reproduce behavior of required axial field profile

\section{Injection Issues at $10 \mathrm{kHz}$ Repetition Rate}

Extrapolation from Table 5 shows that the circulating current at injection is now down to 110 and $240 \mu \mathrm{A}$-ion in FFAG-1 and FFAG-2 respectively. But to take advantage of the HNJ method, the only possible mode of acceleration at such large repetition rate, the beam at injection has a shorter duration than the actual revolution period by the ratio $\beta_{2} / \beta_{1}$ as we discussed earlier. Thus the actual average current to be delivered from the FE is larger by the same factor; this corresponds to an average local injected current into either FFAG ring of $440 \mu \mathrm{A}$-ion.

It may still be possible to perform betatron stacking in a separate Accumulator Ring placed in the same enclosure of both FFAG rings. Table 7 replaces Table 6 with the variation that there are now only $n=20$ turns to be injected in the FFAG-1 case, and 
there is still adequate time for $n=40$ turns in the FFAG-2 case. But this time each turn lasts only $0.96 \mu \mathrm{s}$. We have continued assuming a normalized full beam emittance at the end of stacking $\varepsilon_{\mathrm{n}}=100 \pi \mathrm{mm}$-mrad which yields the same magnet physical gap. Of course the Accumulator Ring is kept at constant energy during the betatron stacking operation, at the end of which the beam is transferred for a single-turn injection into the corresponding FFAG ring. The single turn intensity $\mathrm{I}_{\mathrm{s}}$ is now somewhat lower but still maybe over what it can reasonably be delivered from the ECR ion source. The beam pulse duration is around $70 \mu \mathrm{s}$ in both cases, and the beam emittance of a single pulse should not exceed the values reported in Table 7. As before also here space charge tune depression is not a limiting factor.

Of course stacking of the beam from the ECR in a subsequent EBIS as shown in Figure 7 is also feasible. The ECR-EBIS-RFQ combination operates now at $10 \mathrm{kHz}$ repetition rate. Thus over a period of $100 \mu \mathrm{s}$ the beam is continuously injected and stored from the ECR into the EBIS. At the end of that period of time $2.63 \times 10^{9}$ ions have been accumulated preserving their original charge states $Q=28-29$. The pulse is then extracted with duration of $0.96 \mu$ s. Correspondingly, the instantaneous beam current in exit from the EBIS is $440 \mu \mathrm{A}$-ion. The full beam normalized emittance should not exceed $100 \pi$ mm-mrad to limit the height of the magnet gap to the values given in Table 7, and not be less than $25 \pi \mathrm{mm}$-mrad to avoid a space charge-tune depression exceeding 0.2 .

Table 7. Betatron Stacking in separated Accumulator Ring at $\mathrm{f}_{\mathrm{rep}}=10 \mathrm{kHz}$

$\begin{array}{lll} & \text { FFAG-1 } & \text { FFAG-2 } \\ A & & \\ \varepsilon_{\mathrm{sn}} / \beta \gamma & 560 \pi \mathrm{mm}-\mathrm{mrad} & 240 \pi \mathrm{mm} \text {-mrad } \\ n & 11.2 \pi \mathrm{mm}-\mathrm{mrad} & 2.4 \pi \mathrm{mm}-\mathrm{mrad} \\ \text { Pulse Duration } & 20 & 40 \\ \text { Gap Duration } & 0.957 \mu \mathrm{s} & 0.957 \mu \mathrm{s} \\ \text { Pulse Frequency } & 2.894 \mu \mathrm{s} & 0.794 \mu \mathrm{s} \\ \eta & 0.2597 \mathrm{MHz} & 0.5710 \mathrm{MHz} \\ \mathrm{I}_{\mathrm{s}} & 40 \% & 40 \% \\ 3 \mathrm{~b}=3\left(A \beta_{\mathrm{V}}\right)^{1 / 2} & 22 \mu \mathrm{A}-\mathrm{ion} & 11 \mu \mathrm{A}-\mathrm{ion} \\ \mathrm{T}_{\mathrm{s}} & 165 \mathrm{~mm} & 105 \mathrm{~mm} \\ & 77 \mu \mathrm{s} & 70 \mu \mathrm{s}\end{array}$

\section{CW Mode of Operation}

By extrapolation, a more convenient and useful mode of operation is the Continuous Wave (CW) mode of operation where the beam is continuously injected, accelerated and transferred to the Target. The continuous injection will require that ions occupy simultaneously all orbits as they move in a spiral way in and out from one ring to the next as shown in Figure 12. We do not foresee any major objection or obstacle to this mode of operation that, after all, resemble very closely the one adopted otherwise in 
Cyclotrons, with the provision that one should guarantee a method for injection and extraction with beam sitting on different orbits with enough separation from each other.

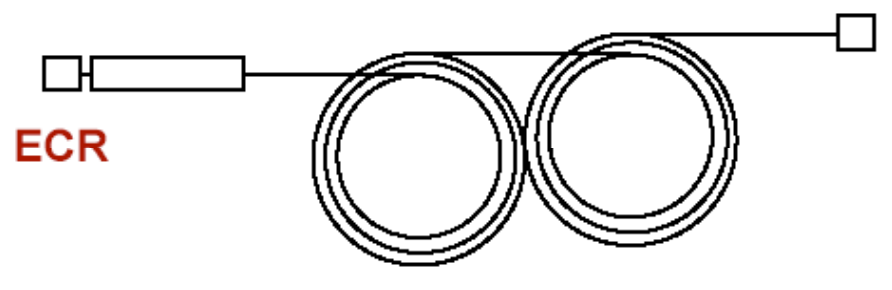

Figure 12. Continuous Mode of Operation of the 2 FFAG rings

The same set up described above and the same RF system, with the HNJ method, can be used with either the single or two FFAG ring scenarios. The required average current on the target is $4.2 \mu \mathrm{A}$-ions. This equals the circulating current at the moment of extraction from the last orbit from the last FFAG ring. This suggests the following mode, shown schematically in Figure 13, of the beam preparation from the FE, going through the Linac section, before entering into the first (or only) FFAG ring. The ECR generates an open sequence of ion pulses at the repetition rate of the injection frequency in either FFAG ring, that is with a periodicity $\mathrm{T}_{\text {initial }}=3.851$ or $1.751 \mu \mathrm{s}$, but each with a duration $\mathrm{T}_{\text {final }}=0.957 \mu \mathrm{s}$ corresponding to the revolution period at the last orbit of the last ring. The average current per pulse is $4.2 \mu \mathrm{A}$-ion. There are 55 bunches in each pulse and the bunching frequency is $57.5 \mathrm{MHz}$, that is there is one bunch every $14 \mathrm{RF}$ buckets at 805 $\mathrm{MHz}$. This time structure is preserved throughout the acceleration cycle, except that the beam pulses get closer to each other and eventually collapse next to each as a real continuous beam on the main Target. The number of ions per turn is a modest $\mathrm{N}_{\text {ions }}=$ $2.51 \times 10^{7}$ and the full beam normalized emittance $2 \pi \mathrm{mm}$-mrad, as extrapolated directly from the SCL approach design. This gives a very modest tune-shift depression. The main $\mathrm{RF}$ and beam parameters for the continuous mode of operation are listed in Table 8 .

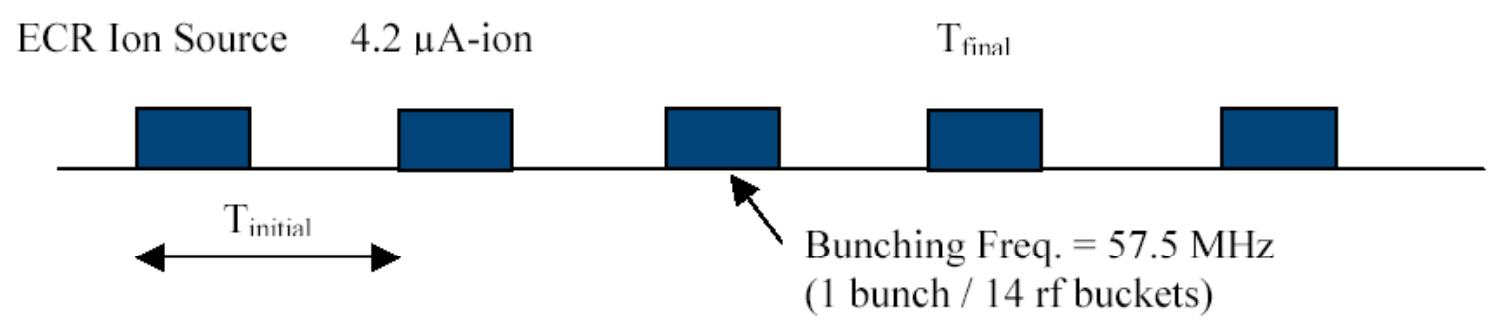

Figure 13. Beam time structure at injection into first FFAG ring

Of course the modality of continuous injection is to be studied, but the ECR ion source alone can certainly provide the required beam intensity. Because of the necessary beam manipulation (chopping, bunching, matching,...) it would be useful if the ECR source could provide a somewhat higher average current, likely close to $10 \mu \mathrm{A}$-ions. 
Because of the fewer number of revolutions and the large beam gap between turns, it is seen, inspecting Table 8 , that the beam width is smaller than the orbit separation at the end of the acceleration cycle that thus allows for a clean single turn extraction. Unfortunately that is not the same situation encountered at injection where the turn separation is not large enough compared to the beam width. Space charge effects are also to be estimated in this multiple turn beam configuration.

Table 8. RF Requirements and Beam Parameters for CW mode of Operation

FFAG-1 $\quad$ FFAG-2

$\begin{array}{lll}\text { Cavity Groups } & 8 & 4 \\ \text { Cavities per Group } & 2 & 4 \\ \beta_{0} & 0.22 & 0.50 \\ \text { Cavity Gap, cm } & 4.1 & 9.3 \\ \text { RF Phase } & 30^{\circ} & 60^{\circ} \\ \text { RF Voltage / Cavity } & 2 \mathrm{MVolt} & 1 \mathrm{MVolt} \\ \text { Single Turn Intensity } & 2.51 \times 10^{7} \text { ions / pulse } \\ \text { Emittance, full normalized } & 2 \pi \mathrm{mm} \text {-mrad } & \\ \text { Orbit Separation, mm } & 0.23-3.4 & 0.30-2.8 \\ \text { Beam rms Width, mm } & 2.2-1.7 & 1.4-1.1 \\ \text { Beam rms Height, mm } & 3.4-3.3 & 2.2-2.2 \\ \text { Space-Charge } \Delta v & 0.028-0.017 & 0.016-0.008\end{array}$

(on a single turn)

\section{Engineering and Cost}

An engineering-like composition that makes one period of the FFAG rings is outlined in Figure 14 that shows the side and top view as well one period with a cryomodule containing a "Cavity" as described earlier and in Figure 11. All the components, magnets, vacuum pipes, flanges, vacuum valves and pumps, steering components and beam diagnostics seem conventional and of relatively easy acquisition. The Cryo-module with the "Cavity" is an exception that requires more involved technical research and design.

It may be too early to make a cost estimate of the project since there are still several technical issues to be studied. But as a simple rule we could reasonably guess that the cost of a period assembled and installed is about $\$ 100$ thousand averaged between the two rings (FFAG-1 could be cheaper by $20 \%$, and FFAG-2 more expensive by also $20 \%$ ). The cost of a cryo-module with the "Cavity" can be estimated around $\$ 500$ thousand. Thus, excluding civil engineering, the cost of FFAG-1 can be around $15 \mathrm{M} \$$, and that of FFAG-2 around $18 \mathrm{M}$. Both of these figures make the FFAG approach competitive with the SCL approach. 


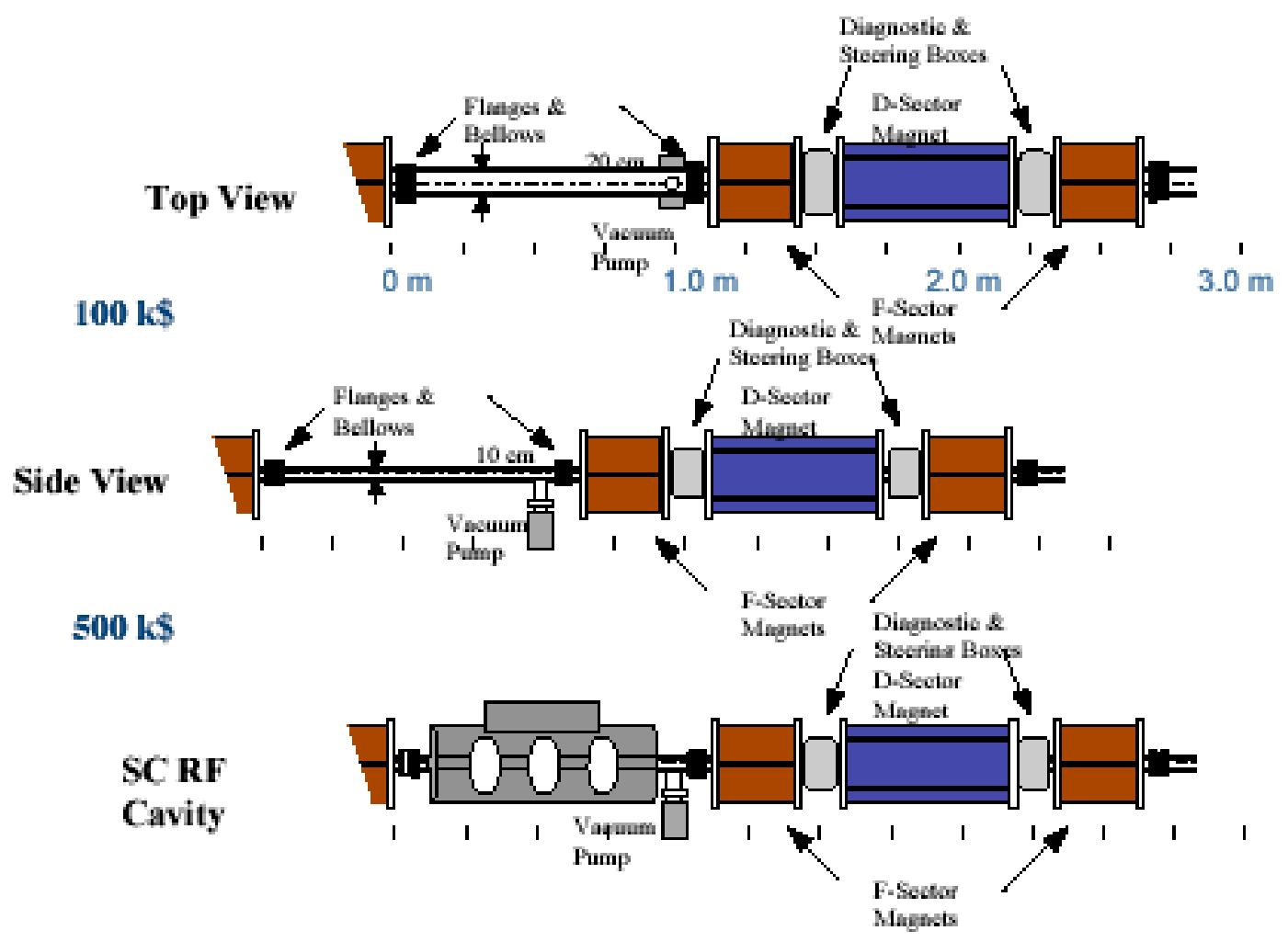

Figure 14. Engineering Layout of a Period

\section{Conclusion}

We have studied the feasibility of acceleration of ions of Uranium 238 in a single or a pair of FFAG rings as an alternative to SCL. The main motivation has been driven of course by possible cost saving. We have investigated three scenarios:

- Operation at $1 \mathrm{kHz}$ repetition rate using Broadband Cavities. Because the limited RF voltage that can be achieved in these cavities, a large number of them is required essentially filling every available long insertion. The power consumption dissipated is also excessive. Otherwise the approach is acceptable. We have encountered problems with storing the required beam intensity because of the limitation of the ion source. Conventional betatron stacking does not seem feasible. A new concept has nevertheless been put forward, that is storage of the beam in an EBIS device following the ECR source. This new concept seems to be quite feasible but of course needs an experimental demonstration.

- Operation at $10 \mathrm{kHz}$ repetition rate using the HNJ. To alleviate the problems with the ion source a higher repetition rate seems required. But acceleration at such large rate cannot be achieved with Broadband Cavities because of the very large number of cavities required. Another method that allows fast acceleration with constant frequency cavities is the Harmonic Number Jump method. Though this method also requires a practical demonstration, it is nevertheless technical feasible 
and appealing. Nevertheless the problem with the ion source is still there though re-dimensioned downward. The ECR-EBIS solution of course still applies and actually it has now a lower performance requirement.

- CW mode of operation. The same HNJ method and the same superconducting cavity layout can also be used with the continuous beam mode of operation. Actually the requirements on the ion source are greatly relieved. There is no need of betatron stacking nor of stacking in the EBIS. The problem remains though of demonstrating the capability of injection and extraction. Maybe a combined operation of stacking the beam for a short period of time and a lower number of simultaneously circulating turns with a larger separation could resolve completely the problems of injection.

\section{References}

[1] P. Ostroumov, Physical Review ST A\&B, 5, 030101 (2002)

[2] AG. Ruggiero, "Brief History of FFAG Accelerators", The Int. Workshop on FFAG Accel., page 9, Dec. 5-9, 2005, KURRI, Osaka, Japan

[3] M. Aiba, "Status of $150 \mathrm{MeV}$ Proton FFAG", The Int. Workshop on FFAG Accel., page 3, Dec. 5-9, 2005, KURRI, Osaka, Japan

[4] M. Tanigaki et al., "Status of FFAG complex at KURRI", The Int. Workshop on FFAG Accel., page 1, Dec. 5-9, 2005, KURRI, Osaka, Japan

[5] The comparison of performance between Scaling Lattice and Non-Scaling Lattice has been documented in several FFAG Workshop, Particle Accelerator Conferences and other meetings during the last several years.

[6] E.D. Courant and H.S. Snyder, Annals of Physics: 3, 1-48 (1958)

[7] A.G. Ruggiero, "Design of Proton FFAG Accelerators", The Int. Workshop on FFAG Accel., page 31, Dec. 5-9, 2005, KURRI, Osaka, Japan

[8] Y. Yamazaky, "Approach to a very high intensity beam at J-PARC", HB2006 Proceedings, Tsukuba, Japan, May 29-June 4, 2006

[9] A.G. Ruggiero, Physical Review ST A\&B 9, 100101 (2006)

[10] S. Henderson, "Recent Beam Commissioning Results from the Spallation Neutron Source", HB2006 Proceedings, Tsukuba, Japan, May 29-June 4, 2006

[11] C. Ohmori, presentation made on the Semiannual 2007 FFAG Workshop, Grenoble, France, April 11-17, 2007, http://lpsc.in2p3.fr/congres/FFAG07/

[12] L. Ahrens et al., "Status and recent performance of the accelerators that serve as gold injector for RHIC", PAC 2001 proceedings, Volume 5, 18-22 June 2001 Page(s):3326 - 3328 